\title{
A SALT Spectral Study of S0s Hosting Pseudobulges
}

\author{
Kaustubh Vaghmare ${ }^{1 \star}$, Sudhanshu Barway ${ }^{2} \dagger$, Petri Väisänen ${ }^{3,4}$, \\ Rajin Ramphul ${ }^{3,5}$, Yogesh Wadadekar ${ }^{6}$, and Ajit K Kembhavi ${ }^{1}$ \\ 1 Inter-University Centre for Astronomy \& Astrophysics (IUCAA), Post Bag 4, Pune 411007, India \\ 2 Indian Institute of Astrophysics, II Block Koramangala, Bangalore 560034, India \\ ${ }^{3}$ South African Astronomical Observatory, P.O.Box 9, Observatory 7935, Cape Town, South Africa, \\ ${ }^{4}$ Southern African Large Telescope, P.O.Box 9, Observatory 7935, Cape Town, South Africa, \\ 5 University of Cape Town, Astronomy Department, Private Bag X3, Rondebosch 7701, South Africa \\ ${ }^{6}$ National Centre for Radio Astrophysics (NCRA), Post Bag 3, Ganeshkhind, Pune 41100\%, India
}

12 July 2021

\begin{abstract}
We present a SALT-RSS spectroscopic study of a sample of S0 galaxies established by Vaghmare et al. (2015) as having pseudobulges using a combination of photometric criteria. We extract the spectra of various regions along the galaxy major axis using standard long-slit spectroscopic reduction procedures and model the spectra using STARLIGHT to derive detailed star formation histories. The central spectra of galaxies without bars in our sample reveal a complex star formation history, which is consistent with the belief that pseudobulges have a history of star formation distributed over extended periods of time. The spectra of the unbarred galaxies contain strong emission lines such as $H \alpha$, indicating active star formation, which appears to be in contradiction with the expectation that S0 galaxies have been stripped of gas. In the case of the two barred galaxies in the sample, the spectrum is dominated by light from a much older stellar population. This seems to suggest an accelerated formation of the pseudobulge made possible by the action of the bar. One of these galaxies appears to have exhausted its reservoir of gas and thus has no signature of a recently formed population of stars while the other galaxy has managed to give rise to new stars through a recent funnelling action. We have also confirmed the influence of bars on the nature of the stellar population in a pseudobulge using an alternate sample based on the SDSS.
\end{abstract}

Key words: methods: data analysis - techniques: image processing - catalogues galaxies: general.

\section{INTRODUCTION}

There are several ways by which one can approach the study of galaxy evolution. One approach is to classify the galaxies in the nearby Universe into different morphological types and study their properties. In such an approach, the study of S0 galaxies has received considerable attention in the last decade. S0 galaxies are characterised by the presence of, what appears to be, an elliptical-like bulge and a smooth outer disk devoid of any apparent spiral arms. Indeed, the study of these galaxies is important because hidden within their inter-relationship with galaxies of other types are clues to the varied processes that play important roles in the overall picture of galaxy evolution (See Aguerri (2012) and references therein).

* E-mail: kaustubh@iucaa.in

† E-mail: sudhanshu.barway@iiap.res.in
For example, observational studies suggest that the central components of S0 galaxies viz. bulges resemble elliptical galaxies (Renzini 1999). They have thus likely formed in a similar manner to ellipticals, i.e. through hierarchical clustering and mergers. However, the observation that the fraction of S0 galaxies within a cluster of galaxies grows at the cost of spirals (Dressler 1980), suggests that S0s originate from spirals. Gas stripping via ram pressure is often considered a viable process for such a transformation in dense cluster environments (Gunn \& Gott 1972; AragónSalamanca et al. 2006; Laurikainen et al. 2010; Maltby et al. 2015). However, we know that S0's are equally common in groups (Wilman et al. 2009; Just et al. 2010; Bekki \& Couch 2011). These observations suggest that the processes such as ram pressure stripping are not responsible for the formation of the majority of S0s in groups where minor mergers and tidal effects can control their evolution (Mazzei et al. 2014; Mapelli et al. 2015). 
Simulations too suggest that there are multiple methods for forming S0s - they can arise in major mergers, can grow through slow/secular processes and can also form through gas stripping of spirals (Querejeta et al. 2015; Tapia et al. 2017). Traces of past merger events which impact the observed kinematics have been observed in some S0s (FalcónBarroso et al. 2004). The observed star formation (SF) and SF history (SFH) characteristics of S0s can be complex (e.g. Barway et al. (2013) and references therein).

Barway et al. (2007, 2009) suggest that the dominant formation process in case of S0s is a function of luminosity, with brighter S0s forming in a manner similar to ellipticals and fainter S0s forming through secular processes or perhaps by gas stripping of spirals (Rizzo et al. 2018), which in turn may have formed through secular evolution. Vaghmare et al. (2013) used archival deep mid-infrared images from the Spitzer Infrared Array Camera to systematically study the bulges of S0 galaxies. Bulges, as defined photometrically, are excesses of light over an inward extrapolation of an exponential profile fitted to the outer disk. They are known to occur in two varieties - classical, resembling ellipticals in almost every aspect including morphology, colour, nature of stellar populations and kinematics, and pseudo, resembling disks and believed to have formed through secular evolution (Kormendy \& Kennicutt 2004). Vaghmare et al. (2013) point out that pseudobulges do exist in S0 galaxies and preferentially occur in fainter S0s. But what is perhaps a more interesting result is that disks of pseudobulge hosting S0s have a lower scale length than those hosting classical bulges.

In Vaghmare et al. (2013), the authors speculate that the lower scale length could imply either (i) that the population of disks with lower scale length on average preferentially host pseudobulges or (ii) that the lowered scale length is a result of some process responsible for either the bulge's or the overall galaxy's growth. In a follow-up study, Vaghmare et al. (2015) compare the disk properties of spirals and S0s and find that pseudobulge hosting S0s have a lower scale length than the pseudobulge hosting spirals. The authors explain this as being due to lowered disk luminosity in case of S0s hosting pseudobulges. They further compare bulge luminosities to demonstrate that only the early-type spirals may transform into S0s with pseudobulges through disk fading via gas stripping, while late-type spirals will need additional processes, especially to aid the growth of bulges to observed luminosities.

The conclusions reached by Vaghmare et al. (2013, 2015) and by Barway et al. rely on statistical arguments. Correlations are derived between various parameters, which in turn are derived from imaging data. While such studies indeed offer insights into broad trends among galaxies in a given class, they cannot provide information on individual galaxies and their star formation / assembly histories. For this, one requires data from multiple wavebands. Barway et al. (2013) use broad band photometry from multiple surveys spanning UV, optical and infrared wavelengths but again use statistical arguments to point out broad trends among S0 galaxies. In order to truly constrain the formation histories of S0s, one needs to have spectroscopic data. Using stellar population synthesis techniques, one can then derive very detailed star formation histories for these galaxies and confront the conclusions reached by the photometric studies. To this effect, we have been using the Southern African
Large Telescope's (SALT) Robert Stoby Spectrograph (RSS) to observe a select sample of S0 galaxies.

The work presented in this paper focusses on a subset of the galaxies - those shown to host pseudobulges by Vaghmare et al. (2015), whose photometric properties and their implications were summarised earlier. The aim here is to check for consistency between star formation histories and the conclusions based on the photometric observations. This paper is organised as follows - the data and the reduction methods are described in section 2 , section 3 explains the process of modelling the spectra using STARLIGHT while sections 4,5 and 6 present the detailed results and possible interpretations.

Throughout this paper, we use the standard concordance cosmology with $\Omega_{M}=0.3, \Omega_{\Lambda}=0.7$ and $h_{100}=0.7$. Also, magnitudes used in this work are in the AB system.

\section{SAMPLE AND OBSERVATIONS}

The sample used in the present study is described in detail in Vaghmare et al. (2013) and Vaghmare et al. (2015). For convenience, we summarise its essential features here. The parent sample comprises $3657 \mathrm{~S} 0$ galaxies as found in the Third Revised Catalogue (de Vaucouleurs et al. 1991). A total B-band magnitude cut-off of 14.0 was imposed to reduce this sample to 1031 galaxies which were then cross-matched with the Spitzer Heritage Archive (SHA). We found midinfrared images taken by the Spitzer Infrared Array Camera (IRAC) for 247 galaxies at $3.5 \mu \mathrm{m}$. These images were processed to achieve the desired quality for $2 \mathrm{D}$ decompositions and structural parameters were obtained by using GALFIT (Peng et al. 2010) for a subset of 185 S0s. Of these, 25 were classified as having pseudobulges using a very conservative criterion - bulges should lie more than 3- $\sigma$ below the Kormendy relation Kormendy \& Kennicutt (2004) and should have an Sérsic index $n<2$. This is a combination of the criteria used by Gadotti (2009) and Fisher \& Drory (2008) (see Vaghmare et al. (2013) for full details).

We used the $10 \mathrm{~m}$ class Southern African Large Telescope (SALT) (Buckley et al. 2006) and its Robert Stobie Spectrograph (RSS)(Burgh et al. 2003; Kobulnicky et al. 2003) to study the sample. The RSS is a versatile instrument providing several observing modes and is designed to work in a wavelength range of $320-900 \mathrm{~nm}$. Of the 25 pseudobulges from Vaghmare et al. (2015), 15 were in the declination range accessible to SALT. We were given time to observe 8 of these galaxies which we present in this paper. A brief summary of the observational parameters is presented in Table 1. Spitzer 3.6 micron imaging for the galaxies is shown in Figure 1.

The long-slit RSS spectra were obtained using a 1" wide slit and the PG0900 grating, placing the slit along the major axis of the galaxy in each case. RSS uses an array of three CCDs of $2048 \times 4096$ pixels, and the grating angle in the instrument was set in a way so as to avoid any important spectral features falling in the resulting two 15 pixel size gaps. We used a $2 \times 4 \mathrm{CCD}$ binning scheme to improve the signal-to-noise ratio (SNR). The spectra obtained in the said configuration have an observed wavelength range of 3640-6765 $\AA$ and thus cover the required optical range for 

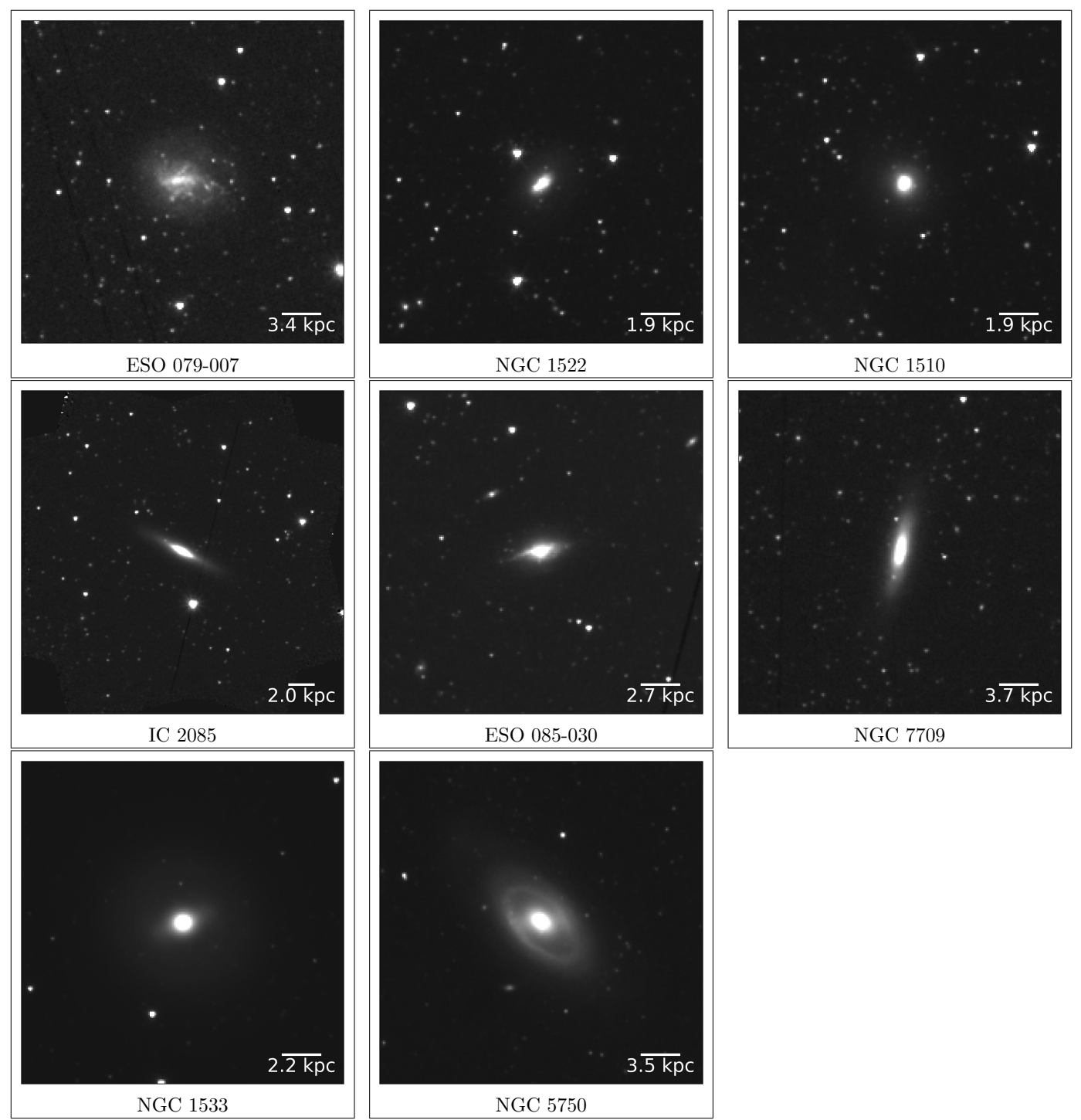

Figure 1. The 3.6 micron images obtained using the Spitzer IRAC, for the eight galaxies studied in the present paper.

Table 1. Basic parameters of the sample galaxies.

\begin{tabular}{lccccl}
\hline Object Name & $\begin{array}{c}\text { RA (J2000) } \\
\text { hh:mm:ss }\end{array}$ & $\begin{array}{c}\text { Dec (J2000) } \\
\text { dd:mm:ss }\end{array}$ & $\begin{array}{c}\text { Total magnitude } \\
\text { B band }\end{array}$ & $\begin{array}{c}\text { Exp. Time } \\
\text { seconds }\end{array}$ & $\begin{array}{l}\text { Position Angle } \\
\text { degree }\end{array}$ \\
\hline ESO079-007 & $00: 50: 04$ & $-66: 33: 10$ & 13.73 & 4500 & 2.6 \\
NGC1522 & $04: 06: 08$ & $-52: 40: 06$ & 13.97 & 5000 & 38.6 \\
NGC1533 & $04: 09: 52$ & $-56: 07: 06$ & 11.79 & 3600 & 170 \\
NGC1510 & $04: 03: 33$ & $-43: 24: 01$ & 13.47 & 4500 & 102.9 \\
IC2085 & $04: 31: 24$ & $-54: 25: 01$ & 13.95 & 5000 & 112 \\
ESO085-030 & $05: 01: 30$ & $-63: 17: 36$ & 13.79 & 4500 & 148.2 \\
NGC5750 & $14: 46: 11$ & $-00: 13: 23$ & 12.55 & 4500 & 70.6 \\
NGC7709 & $23: 35: 27$ & $-16: 42: 18$ & 13.60 & 4500 & 55 \\
\hline
\end{tabular}

Notes: Column 1 is the galaxy name ; Column $2 \& 3$ are Right Ascension and Declination of galaxy ; Column 4 is the total B band magnitude; Column 5 is the total exposure time for RSS spectrum and Column 6 is the slit position angle, measured North to East. 
unravelling the stellar formation histories of these galaxies. The spectral resolution is $\sim 3 \AA$.

We used multiple 600-900 sec individual exposures for achieving the total exposure times per galaxy as listed in Table 1 . For 7 of the 8 galaxies in our sample, this was done over two separate visits to the targets. Calibration unit Arc lamps and flat-field images were observed with the same configuration as used for the science targets and spectrophotometric standards.

\subsection{Spectroscopic Reduction}

We made use of the SALT product data generated by the inhouse pipeline called PySALT (Crawford et al. 2010), which mosaics the individual CCD data to a single FITS file, corrects for cross-talk effects, and performs bias and gain corrections. We then carried out further reduction steps using our own custom tools, written in Python/PyRAF, which consist of several modules designed to handle specific steps of processing. First, we trim the regions of the CCD not containing any usable data and then fill the CCD gaps using a gradient fill. To do this, we calculate the the gradient across the gap by measuring the flux values of the pixels to the right and the left of the gap, computing the difference and normalising it to the number of pixels belonging to the CCD gap (the gradient). We then use the gradient to determine the flux values for pixels in each row of the image and replace the zero value pixels by these flux values. This step is important to prevent discontinuities in the brightness distribution which affect the flat-fielding process which relies on the modelling of the large scale illumination structure. Another advantage of this gradient fill is seen in the subsequent step of background subtraction. A CCD gap which appears straight in the original images becomes curved as a result of the coordinate transformation step. This causes background subtraction to result in discontinuous patches. By filling the CCD gap, these patches can be avoided. We then remove the cosmic rays using the LACosmic algorithm (van Dokkum 2001). Calibration in wavelength is achieved using arc lamps and has typical error of $\pm 0.35 \AA$. We use the arc lamp to determine a coordinate transformation to 'straighten' frames so that every column corresponds to a unique wavelength making the background sky shape fitting and subtraction possible. Using the spectrophotometric standard data, we determine the relative flux calibration. We then check individual frames for alignment and co-add them. During this step, a standard deviation frame is constructed from which an error frame is obtained. Before the co-addition, to have consistent noise characteristics, we equalize the effective exposures of the frames - this is needed because the pupil of SALT changes by design during the observation. If $O_{i}(x, y)$ are the individual observed frames, the final spectrum $F(x, y)$ and error spectrum $E(x, y)$ can be written as,

$F(x, y)=\langle O(x, y)\rangle=\frac{\sum_{i}^{N} O_{i}(x, y)}{N}$

$E(x, y)=\sqrt{\frac{\sum_{i}^{N}\left(O_{i}(x, y)-\langle O(x, y)\rangle\right)^{2}}{N-1}}$
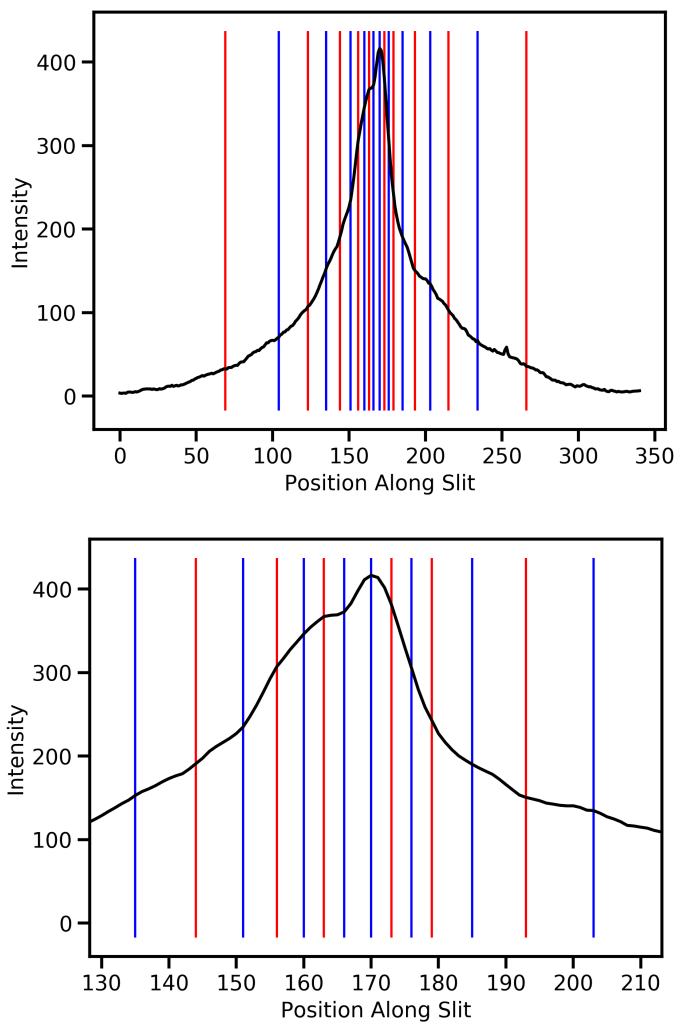

Figure 2. A plot of the brightness profile of a galaxy along the slit. The apertures, rows which are summed up to produce the $1-\mathrm{d}$ spectrum in the given region of the galaxy, are marked in alternating colors. The rows lying between a pair of successive red and blue lines are summed to obtain the spectrum corresponding to the centre of this aperture. As can be seen, the width of these apertures increases in inverse ratio to the level of flux. The lower panel shows a close-up of the central region.

An alternate way of obtaining the error frames is to start from the raw CCD images whose noise properties are relatively well understood to try to model the effects of every single reduction step on the errors. This is however very complicated and so we adopt the above technique of determining our final error frame. Since we are combining only 6 - 8 frames, we might overestimate our errors, but this over-estimation does not affect the findings presented in the paper. Our final step is to correct for foreground extinction and this is done using the deredden task in IRAF.

\subsection{Post Reduction Procedure}

The modelling of the spectrum to study its star formation history has been done using STARLIGHT (Cid Fernandes et al. 2005). This program requires a 1-d spectrum in the form of an ASCII table comprising at least two columns, namely wavelength and flux. Furthermore, we wish to study the spectra at different spatial positions. We thus extract 1-d spectra from positions along the long slit by summing up a suitable number of rows. If this number is kept constant as a function of the position on the long slit, the signal-to-noise ratio degrades for spectra extracted far away from the centre due to the decreased brightness of the galaxy in this region. 
So, we came up with a scheme of increasing the number of rows summed up as an inverse function of the total amount of flux from the galaxy in a given region of the slit. This is illustrated in Figure 2, which shows the flux in scaled counts as a function of position along the slit. The routine that determines the sizes of these apertures requires as an input the number of apertures to be extracted and the range of the spatial intensity profile over which the extraction is to be carried out, and then determines these aperture sizes in units of pixels so as to keep the total flux within the aperture roughly constant. The routine does not take into account the physical scale of the galaxy. The number of apertures to be extracted are chosen subjectively based on the overall quality of the spectrum which in turn depends on the brightness of the galaxy.

Due to the rotation of stars in the galaxies, the spectra represented by individual rows are shifted with respect to each other. We constructed a Python based module to characterise this by tracing the centroid of individual absorption features along the long slit. A rotation curve of the galaxy is thus obtained. By fitting polynomials of suitable order to this curve, it is also possible to derotate individual rows for the purpose of stellar population fitting - this is necessary to not introduce broadening of spectral features due to rotation. We shift each row in the wavelength space using the fitted rotation curve information before co-adding rows to be fit with STARLight. During the coaddition of the rows, the corresponding rows from the error frame are also added in quadrature and divided by number of rows to get the error spectrum. The spectrum and the related uncertainties can now be read and modelled by STARLIGHT.

\section{SPECTRAL MODELLING}

There are several methods to derive the star formation history of a galaxy from its spectrum (see Sánchez-Blázquez (2016) for a critical summary). In the present study, we adopt the method implemented in STARLIGHT (Cid Fernandes et al. 2005). We briefly explain the method for the convenience of the reader.

Any arbitrary star formation history can be approximated as a series of star bursts in each of which a population of stars with a given initial mass function (IMF) is formed almost at the same time. The spectrum of such a population, known as a Simple Stellar Population (SSP), can be determined as a function of age and metallicity, knowing the IMF and adopting a stellar spectral library. The observed spectrum can then be modelled as a linear combination of spectra of different SSP. A galaxy whose formation is consistent with a single episodic burst of star formation would be described by a single SSP and thus any attempt to model such a galaxy spectrum would lead us to finding virtually no contribution from any SSP but one. And for any other formation history, the relative contributions would be proportional to the strength of the star bursts at each epoch. This way, the star formation history of the galaxies can be obtained.

Such a method is implemented by STARLIGHT which accepts an input 1-d spectrum, a set of base spectra and data flags/masks and fits a population vector to the observed spectrum, which tells us the relative contribution of each spectrum in the base to the observed spectrum. Mathematically, the model spectrum can be written as

$M_{\lambda}=\sum_{j=1}^{N_{*}} P_{j} L_{\lambda, j}^{0} * G\left(v_{*}, \sigma_{*}\right) 10^{-0.4 A_{\lambda, j}}$

Here, $P_{j}$ refers to fractional contribution of jth base spectrum to the total spectrum. $L_{\lambda, j}^{0}$ refers to the jth base spectrum without any extinction or kinematic filters applied, G refers to a Gaussian characterized by a centroid $v_{*}$ and a width $\sigma_{*}$. The operation $*$ implies a convolution. The last multiplicative factor is to account for intrinsic extinction along the line of slight. $N_{*}$ refers to the total number of base spectra.

However, an important issue concerning the use of STARLIGHT is with regards to the base selection. A hand picked base may be biased towards certain ages and metallicities and thus introduce biases in the deduced star formation history. An attempt to conservatively choose all possible ages and metallicities increases computation overheads (as $N_{*}^{2}$ ). We thus adopt the prescription of Richards et al. (2009) who suggest using diffusion mapping with K-means clustering to obtain a base of $\mathrm{M}$ spectra starting from a very large number $\mathrm{N}$ of base spectra covering all or most of the parameter space of interest, with $M \ll N$. For the present study we obtain 150 base spectra from the MILES (Sánchez-Blázquez et al. 2006) spectral library obtained using the Salpeter IMF, and use diffusion mapping with K-means clustering to construct a base of 45 spectra, which uniformly covers the agemetallicity parameter space. The initial base spectra are obtained from a web application provided by the MILES group. The code for applying the Richards et al. (2009) algorithm is provided by them as a MATLAB code. We wrote a converter to reformat the spectra obtained from the MILES site into a form compatible with the MATLAB code. We wrote another script in Python to transform the output of the MATLAB code into a form compatible with STARLIGHT. The final 45 spectra used for modeling the spectra of the galaxies is presented in Appendix A.

In order to compute the errors on the parameters returned by STARLIGHT, we adopt the following approach. We use the derived error spectrum to construct several $(\sim 100)$ realisations of the observed spectrum and run STARLIGHT for each one of them. These realisations are constructed by approximating the underlying error distribution to be a Gaussian and drawing random samples from a Gaussian distribution with zero mean and a standard deviation equal the corresponding uncertainty read off from the error spectrum. The standard deviation of any given parameter obtained for these realisations is adopted as an estimate of its uncertainty. As noted earlier, the method by which error frames are being constructed is likely to lead to an over-estimation of the uncertainty. This means that the error bars obtained on the STARLIGHT parameters will also be over-estimated. As can be gauged from the discussion of the science results in the subsequent sections, this over-estimation does not affect the conclusions drawn in this paper. 


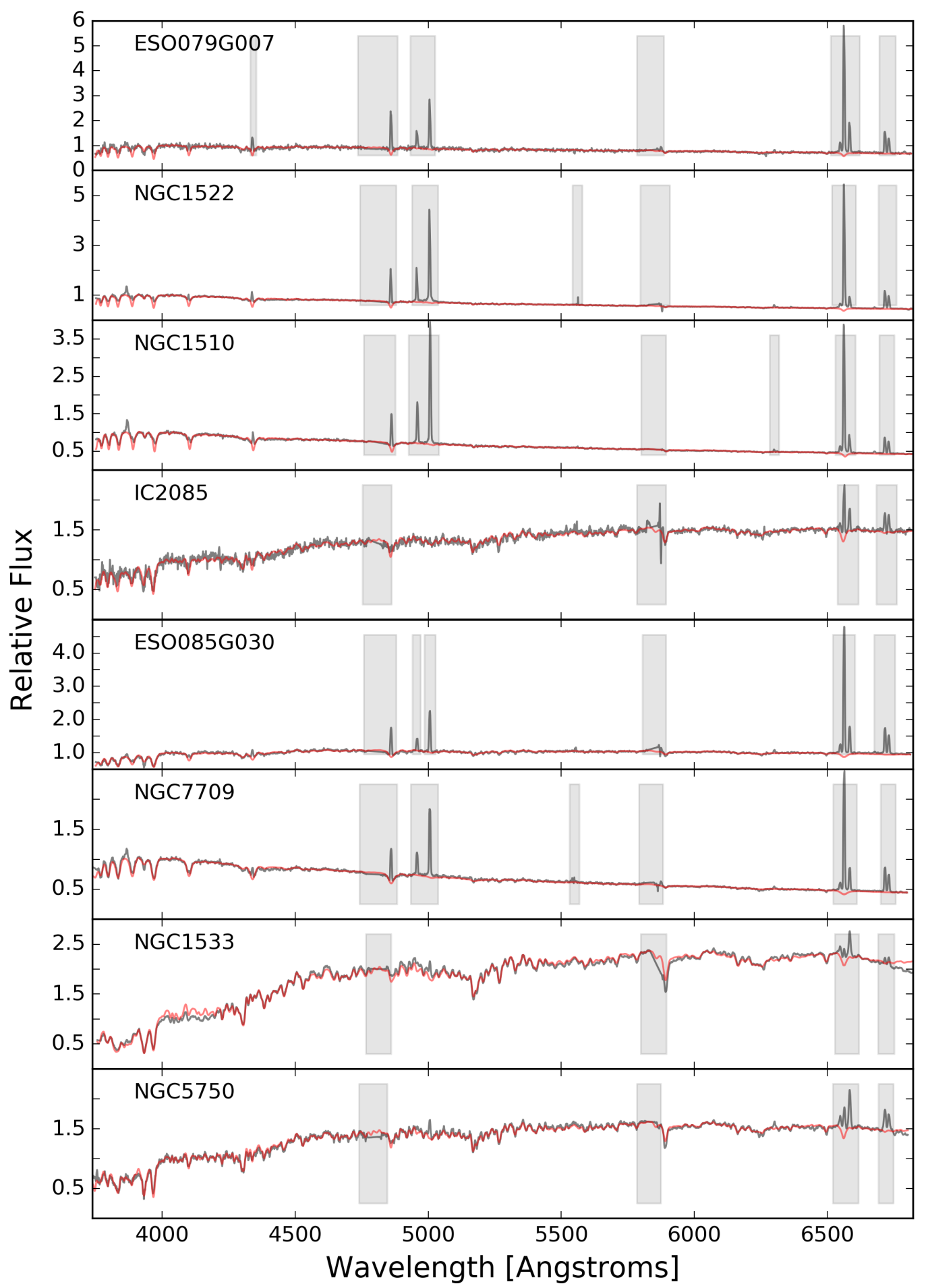

Figure 3. Observed spectrum (grey line) and best-fit STARLIGHT model (red line) for the central regions of galaxies in our sample. The grey regions indicate wavelengths ignored during the STARLIGHT fit as they contain emission from hot gas or are lost in the CCD gaps. 


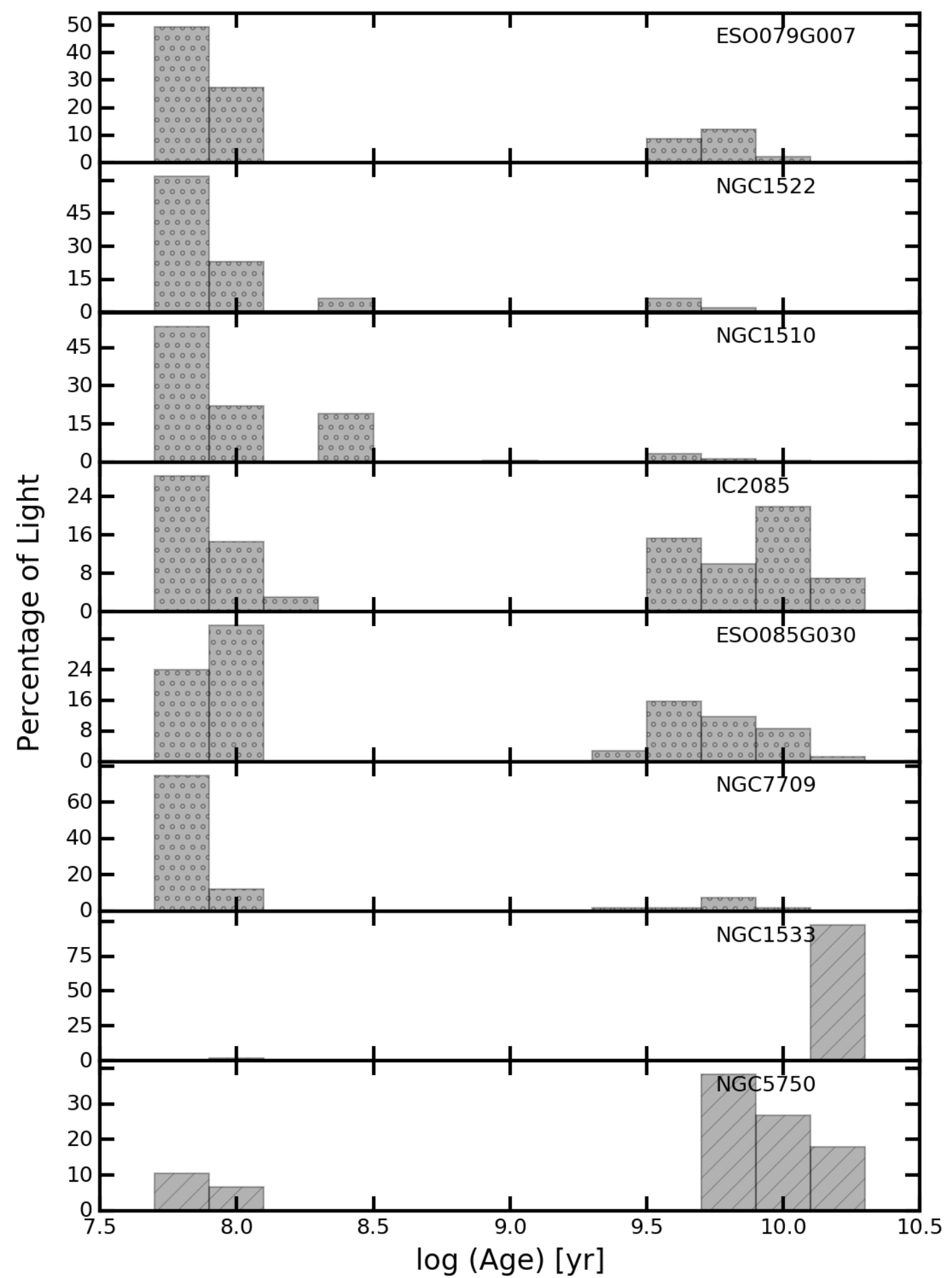

Figure 4. Star formation histories for the galaxies in our sample. The $\mathrm{X}$-axis indicates the logarithm of the age and the Y-axis the percentage of light contributed to the observed spectrum by a stellar population of the given age. These percentages are computed from the population vector returned by STARLIGHT. The hatch patterns are circles for the first six galaxies which are unbarred and forward leaning diagonals for the last two galaxies which are barred. 


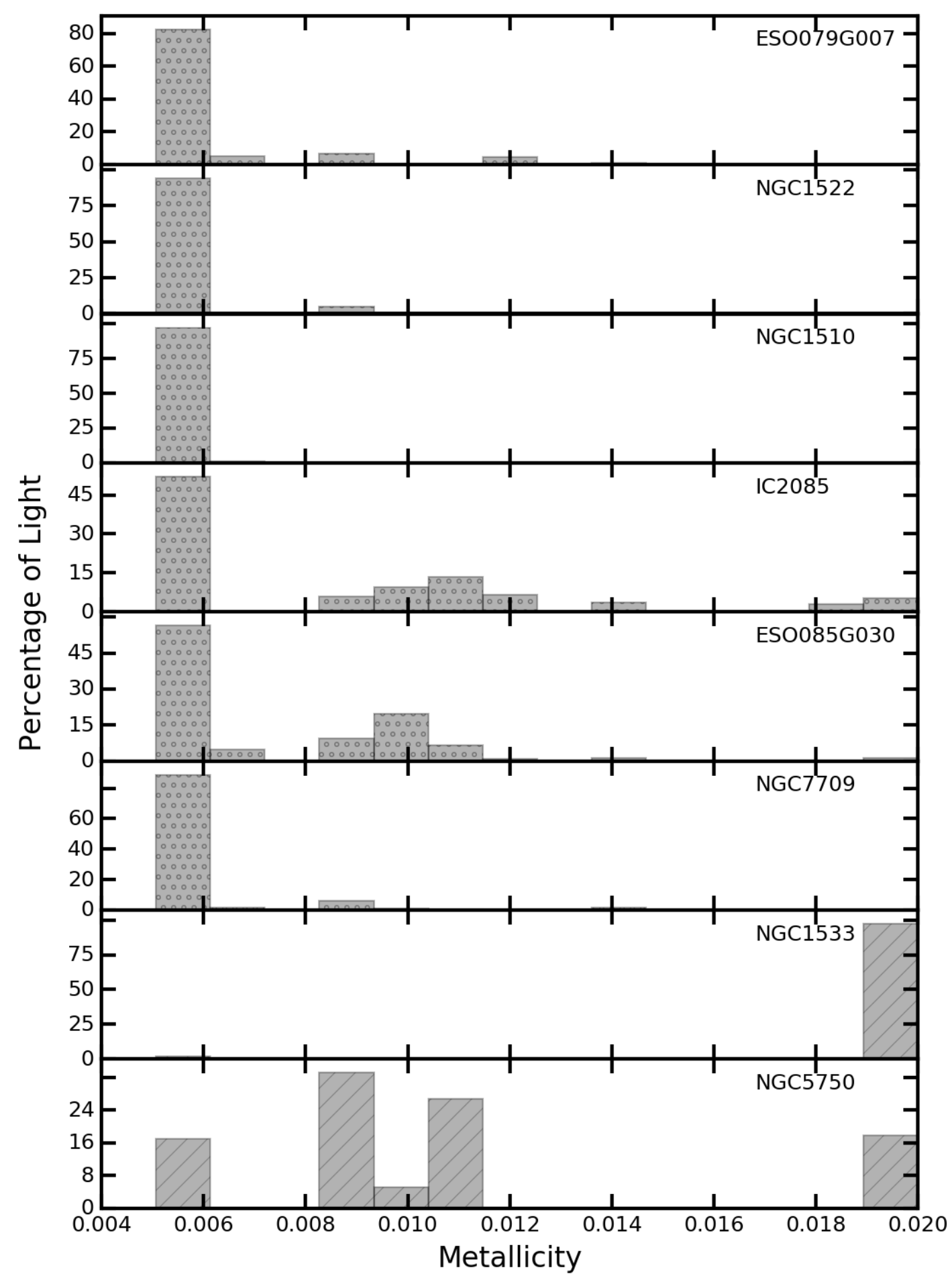

Figure 5. Metallicity contributions for the galaxies in our sample. The $\mathrm{X}$-axis is the metallicity while the Y-axis represents the fractional contribution to the flux of the observed spectrum as determined from the population vector. The hatch patterns are circles for the first six galaxies which are unbarred and forward leaning diagonals for the last two galaxies which are barred. 

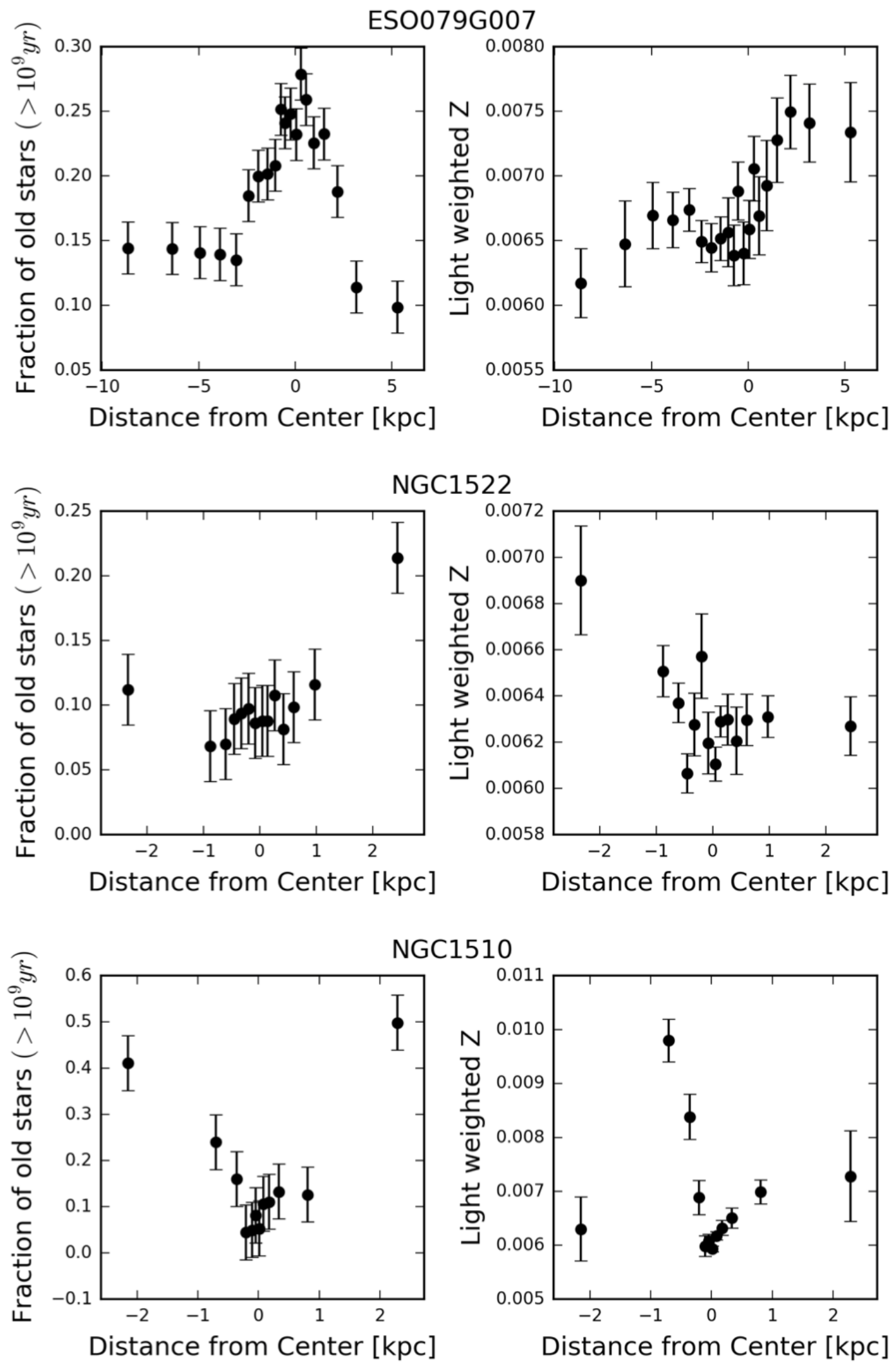

Figure 6. Age and metallicity gradients for the galaxies in our sample. The X-axis in all the plots is the distance relative to the centre of the galaxy in kpc. For plots on the left, the Y-axis indicates the fraction of the stars with age $>10^{9}$ yr, while for the plots on the right, it indicates the light weighted metallicity. Both quantities are computed by STARLIGHT and can be obtained from the population vector output by it. 


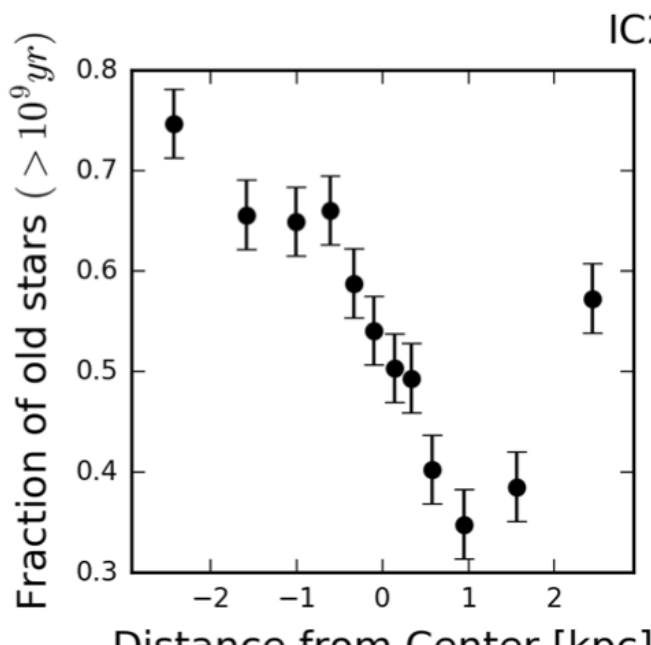

IC2085

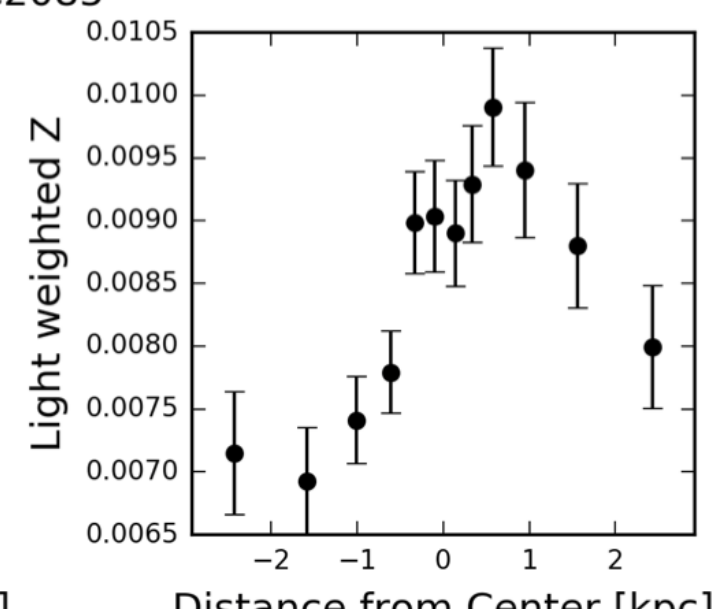

Distance from Center [kpc]

Distance from Center $[\mathrm{kpc}]$
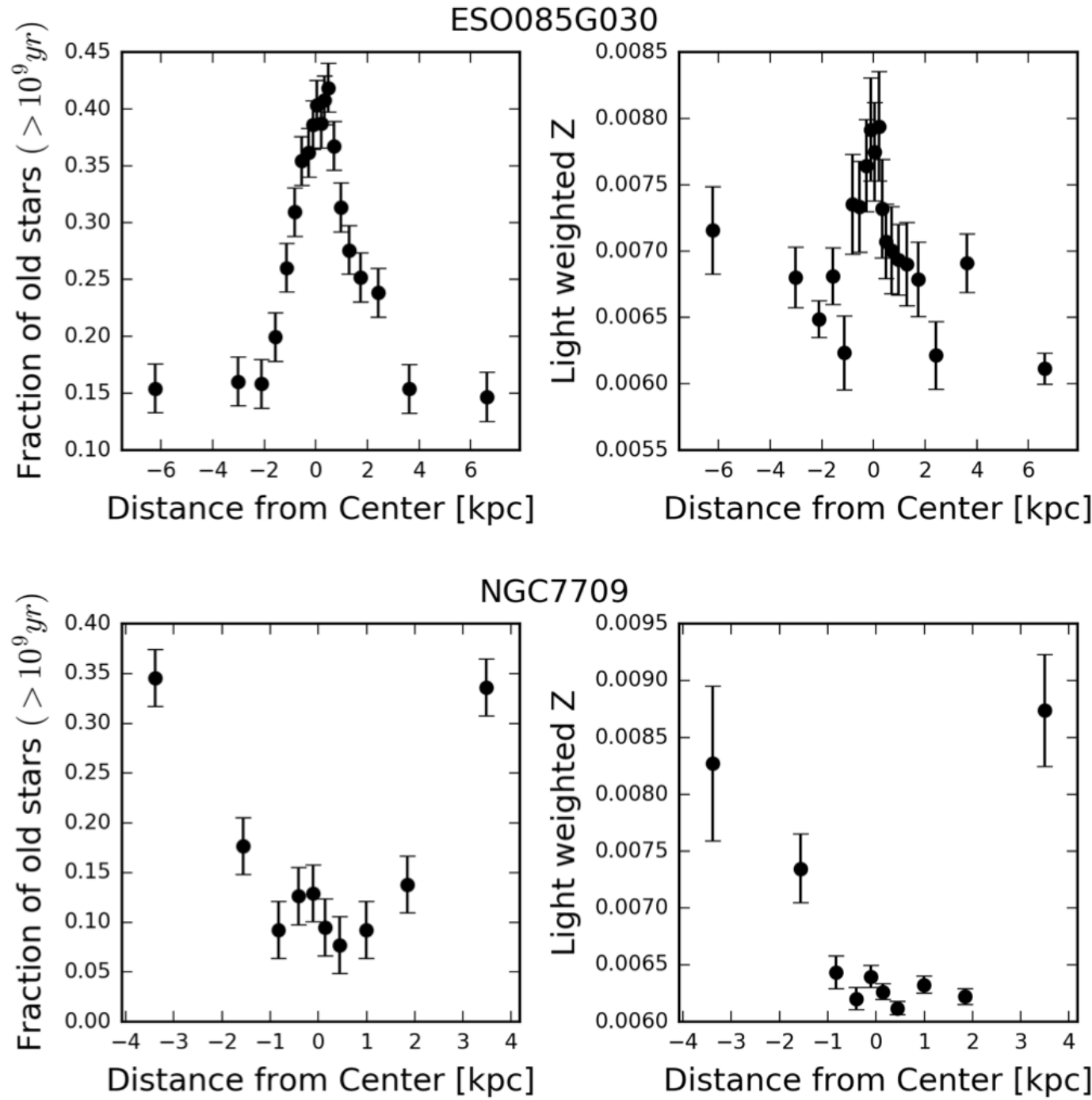

Figure 6 - continued Age and metallicity gradients for the galaxies in our sample. The X-axis in all the plots is the distance relative to the centre of the galaxy in kpc. For plots on the left, the Y-axis indicates the fraction of the stars with age $>10^{9}$ yr, while for the plots on the right, it indicates the light weighted metallicity. Both quantities are computed by STARLIGHT and can be obtained from the population vector output by it. 

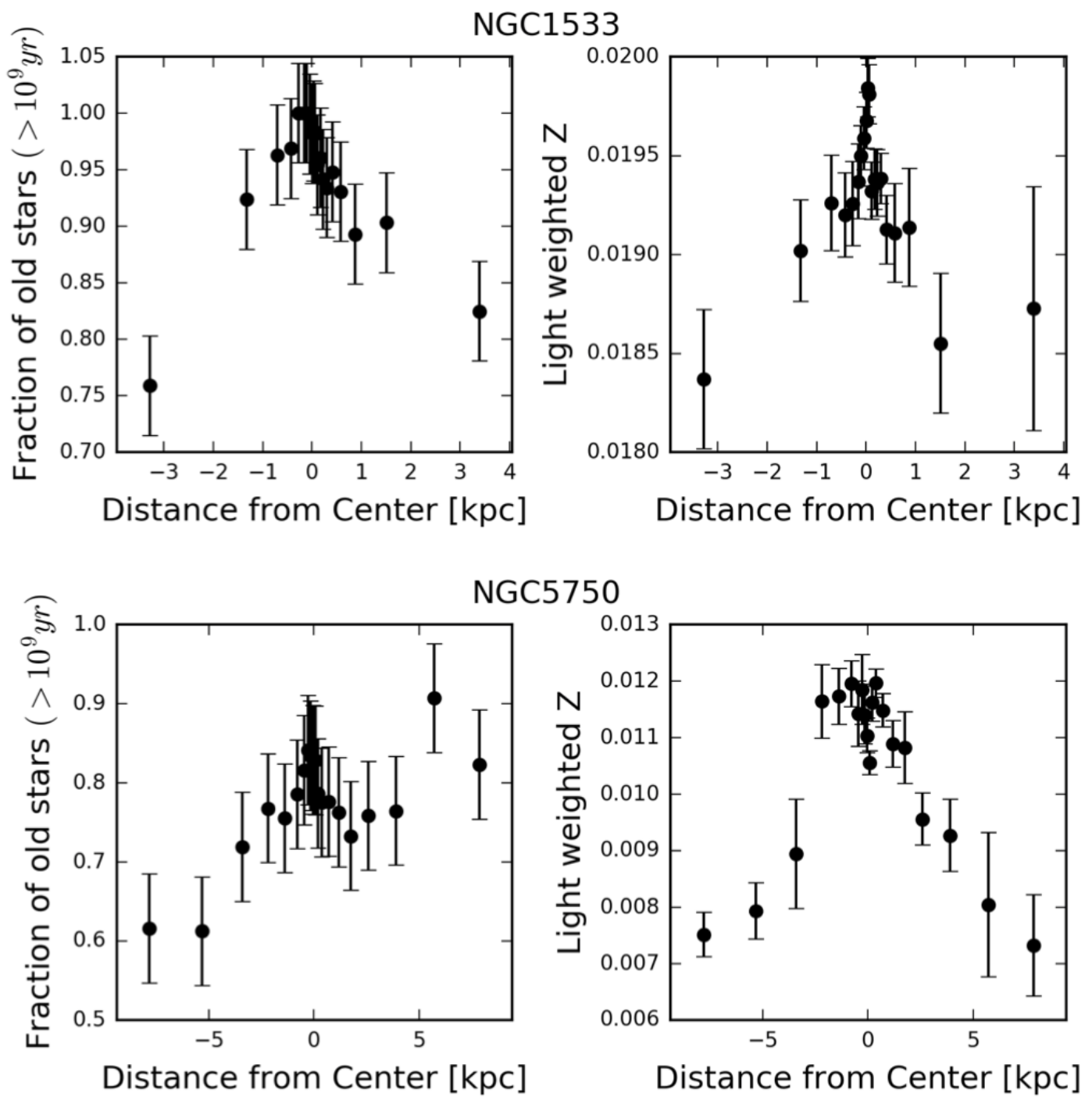

Figure 6 - continued Age and metallicity gradients for the galaxies in our sample. The X-axis in all the plots is the distance relative to the centre of the galaxy in kpc. For plots on the left, the Y-axis indicates the fraction of the stars with age $>10^{9}$ yr, while for the plots on the right, it indicates the light weighted metallicity. Both quantities are computed by STARLIGHT and can be obtained from the population vector output by it.

\section{RESULTS}

For the eight galaxies in the current sample, we have the photometric parameters from Vaghmare et al. (2015) obtained by modelling the mid-infrared surface brightness distribution. These parameters are reproduced in Table 2. In Table 3 , we have various spectral parameters derived by fitting STARLIGHT models to the spectra from the central regions of these galaxies. Also included in this table are $D_{n}(4000)$ index (Balogh et al. 1999) values computed as the ratio of the integrated fluxes from $4000-4100 \AA$ and $3850-3950 \AA$ bands. Using standard statistical techniques, we have studied correlations among photometric and spectroscopic parameters which we discuss in Section 4.3.

Using the tools and techniques explained in the previous sections, we were able to model the spectra from the central regions of the galaxies in our sample and derive their various stellar population parameters. The central spectra and the best fit STARLIGHT models are shown in Figure 3. The star formation history as output by STARLIGHT is a population vector which contains percentage contributions of SSPs of various ages and metallicities, present in the base spectra, to the observed spectrum. To represent the star formation history of the galaxy on a plot, we consider uniform bins of age in the log space and use the population vector to determine the total percentage contribution by all SSPs in a given age bin. So the plot is made with the logarithm of the age on the $\mathrm{X}$ axis and percentage of light contributed by an $\mathrm{SSP}$ at that age along the Y-axis. The star formation histories of the galaxies are shown in Figure 4 and the metallicity histograms in Figure 5. 
Table 2. Best fit Bulge and Disk parameters for the S0 galaxies in our sample.

\begin{tabular}{lcccccccccccc}
\hline Name & $T$ & \multicolumn{3}{c}{$L_{K}$} & \multicolumn{3}{c}{ Bulge parameters } & \multicolumn{3}{c}{ Disk parameters } & \multicolumn{3}{c}{ Bar Parameters } & \multicolumn{2}{c}{$\mathrm{B} / \mathrm{T}$} & Bar/T \\
& & & \multicolumn{2}{c}{$<\mu_{e}>$} & $\mathrm{r}_{\mathrm{e}}$ & $n$ & $\mu_{0 d}$ & $\mathrm{r}_{\mathrm{d}}$ & $\mathrm{m}_{\text {bar }}$ & $n_{\text {bar }}$ & $\mathrm{r}_{\mathrm{e}(\mathrm{bar})}$ & \\
\hline \hline ESO079-007 & -2.0 & -18.73 & 23.25 & 0.87 & 0.53 & 21.23 & 1.72 & - & - & - & 0.04 & - \\
NGC1522 & -2.3 & -18.33 & 20.63 & 0.36 & 0.44 & 21.83 & 0.81 & - & - & - & 0.37 & - \\
NGC1510 & -1.8 & -20.24 & 19.18 & 0.24 & 1.18 & 21.41 & 0.82 & - & - & - & 0.40 & - \\
IC2085 & -1.2 & -18.48 & 20.37 & 0.53 & 0.88 & 21.79 & 1.24 & - & - & - & 0.41 & - \\
ESO085-030 & -0.4 & -18.76 & 20.50 & 0.86 & 1.41 & 20.94 & 0.78 & - & - & - & 0.64 & - \\
NGC7709 & -1.9 & -20.92 & 20.65 & 0.72 & 0.41 & 21.03 & 1.70 & - & - & - & 0.20 & - \\
NGC1533 & 0.1 & -18.79 & 16.73 & 0.22 & 0.95 & 19.77 & 1.58 & 12.94 & 0.50 & 0.89 & 0.22 & 0.07 \\
NGC5750 & -2.0 & -21.62 & 17.84 & 0.32 & 0.98 & 19.94 & 2.56 & 13.64 & 0.20 & 2.23 & 0.09 & 0.09 \\
\hline
\end{tabular}

Notes: Column 1 - The common name of the galaxy; Column 2 - Hubble stage parameter T, Column 3 - K-band absolute magnitude (AB system), Column 4 - the average surface brightness of the bulge within its effective radius, in mag per arcsec ${ }^{2}$, Column 5 - bulge effective radius in kpc, Column 6 - the bulge Sérsic index, Column 7 - disk central brightness in mag per $\operatorname{arcsec}^{2}$, Column 8 - disk scale length in kpc, Column 9 - integrated apparent magnitude of the bar, Column 10 - Sérsic index of bar, Column 11 - Bar effective radius in kpc, Column 12 - Bulge-to-Total luminosity ratio, Column 13 - Bar-to-Total luminosity ratio.

In Figure 6, we show the age and metallicity gradients for the six unbarred galaxies and the two barred galaxies. These plots are constructed as follows. For any given galaxy, we define the the center using light weighted average row number for the brightness profile along the slit. This is labelled as zero in all the plots. For each spectrum derived, a light weighted centroid is assigned to it using the spatial profile in the series of rows which were summed up to obtain the spectrum. STARLIGHT models give us light weighted age and metallicity for this spectrum as well as the errors on them. This way, one can obtain the gradients of the light weighted age as well as the light weighted metallicity.

\subsection{Broad Trends}

In this section, we will discuss the broad trends found in this sample of eight galaxies and reserve the next section for comments on individual objects. As discussed earlier, the galaxies in this sample are a subset of the sample of photometrically classified S0s hosting pseudobulges from Vaghmare et al. (2015). Pseudobulge hosting galaxies are in general expected to have a complex star formation history viz. their spectrum cannot be described using that of a Simple Stellar Population. This is because pseudobulges are believed to have been formed through slow and secular processes with sustained star formation over a long period of time. An SSP, on the other hand, describes a stellar population which has taken birth in a single episodic burst.

The photometric studies on the current sample done by Vaghmare et al. (2015) reveal that their disks have a smaller scale length than their counterparts among spiral galaxies. The authors argue that since the total luminosity of the disk goes as $L \sim I_{d}(0) r_{d}^{2}$ the smaller scale length $r_{d}$ implies that disks have a lower luminosity, assuming similar values of $I_{d}(0)$ which they observationally confirm. They argue that this lower luminosity is due to stripping of gas, a process often invoked to explain the fading of spiral arms in spiral galaxies resulting in a morphological transformation to S0s. The authors further argue in favor of gas stripping of spiral disks by comparing bulge luminosities. Pseudobulges in S0s have a lower luminosity, which they say is due to the lower availability of gas in S0s to drive bulge growth.
Based on these two known aspects of the current sample of S0s - that they are pseudobulge hosts and they have undergone gas stripping, one would expect two results from a detailed spectral study of such galaxies. Firstly, we expect the spectrum of these galaxies to not be well fitted by a single SSP spectrum. Secondly, we expect no strong indications of recent star formation. Both these expectations can now be tested using the spectral data obtained in the present study.

In Figure 3 we show the final reduced spectra from the central regions of the eight galaxies. It is clear that the spectra of the non-barred galaxies (first six) indicate a population dominated by young and recently formed stars while the spectra of barred galaxies indicate an old and evolved stellar population likely to be well described using an SSP. A spectrum of a typical elliptical galaxy, a class of galaxies known to be consistent with having an SSP, are devoid of strong emission lines and exhibit a strong break at the 4000 $\AA$ region due to metal absorption in the outer atmospheres of old stars. Such a break is seen in the spectrum of the two barred galaxies. But the break is absent from the spectrum of five of six unbarred galaxies in Figure 3, indicating that the spectrum in their case is dominated by contributions from a younger stellar population.

Further, one can see that most of the spectra show strong emission lines such as $H \alpha$. This is counter to the expectation that there should be no ongoing star formation in the current sample of S0 galaxies, assuming that they are fully gas stripped and transformed spirals.

In Figure 4, we see the derived star formation history of the central regions of these galaxies. One can again see that the barred and the unbarred galaxies behaving differently. In case of unbarred galaxies, we see peaks towards the lower end of the age axis indicating that a large fraction of the light contributed to the observed spectrum comes from the lower age population. We see the opposite result in the case of barred galaxies which show higher peaks at the higher end of the age axis. An important point to note regarding Figure 4 concerns the lower limit on the age axis. The strong $H \alpha$ lines suggest there must be a population of stars with $\log ($ age $)<7.0$ but the lowest point on the age axis is 7.5. This is because the MILES spectral library does not allow 


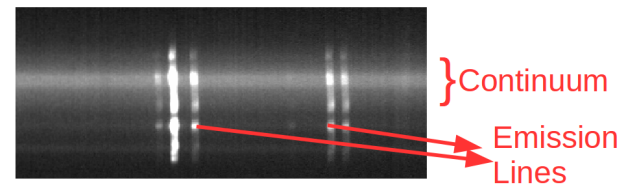

Figure 7. A narrow region of the 2-d spectrum of the galaxy ESO079-003, near the $H \alpha, \mathrm{N}[\mathrm{II}]$ and S[II] emissions. The continuum has been marked along with the emission lines. As can be seen, the distribution of the emission lines about the continuum is asymmetric.

one to probe ages lower than this and not necessarily because there is no population of age lower than the above limit. So, the lowest age bin should be interpreted as containing percentage contribution from populations at that age and lower.

Next, we explore the metallicity distributions in the unbarred and barred galaxies, as shown in Figure 5 respectively. Again, it is easy to spot the differences. In case of unbarred galaxies, there is a common peak at $0.005-0.006$ subsolar metallicity with little contribution from higher metallicities. On the other hand, in case of barred galaxies, metallicities of different ranges have comparable contributions to the stellar populations.

Having summarised the broad trends in spectra, the star formation histories and the metallicity distributions of the galaxies in the current sample, we now move towards a more detailed description of individual galaxies.

\subsection{Discussion of Individual Objects}

\subsubsection{ESO079-007}

The NASA Extragalactic Database (NED), describes this S0 galaxy as being peculiar. What sets this galaxy apart from conventional S0 galaxies is the presence of asymmetric star forming regions i.e. the star forming regions are more towards one side of the centre than the other. To illustrate this better, we show in Figure 7, the 2-d spectrum near the wavelength region dominated by $H \alpha, \mathrm{N}[\mathrm{II}]$ and $\mathrm{S}[\mathrm{II}]$ emission lines. The figure shows the stellar continuum in the centre of the galaxy. It is easy to see that there is a stronger star formation activity on one side of the galaxy centre than the the opposite side. The overall metallicity of the galaxy is also sub-solar.

It is likely that the galaxy is accreting gas only from one direction in the disc which builds up the gas reservoir to induce disc instabilities and trigger asymmetric star formation (Vulcani et al. 2018). Galaxy interactions in the form of fly-bys or recent accretion of smaller satellites could also account for the asymmetric distribution of star formation within the disc (Mapelli et al. 2008).

\subsubsection{NGC 1522}

NED classifies this galaxy as a peculiar S0 galaxy. The spectrum of this galaxy suggests a very young population and strong ongoing star formation dominating the light budget at optical wavelengths. STARLIGHT models suggest that $95 \%$ of the flux is explained by a stellar population younger than
$10^{8} \mathrm{yr}$. What makes the object even more interesting is that it is highly metal poor suggesting that the gas responsible for the ongoing star formation is not enriched by any Population 2 stars.

Bulge-disk decomposition for this galaxy shows that the bulge component of the stars accounts for $40 \%$ of the total galaxy light and the rest by a faded disk. Yet again we have a case of a galaxy whose star formation indicates availability of a large amount of gas but the disk shows signs of having faded, assuming a spiral progenitor with a brighter disk.

\subsubsection{NGC 1533}

This S0 galaxy is a part of the Dorado Group with a clearly discernable bar structure. It exhibits a smooth appearance and one look at the spectrum of the galaxy reveals that it is dominated by a very old population. An age gradient plot reveals that indeed, $95 \%$ of the galaxy light is dominated by old stars. From 3.6 micron imaging, we know the bulge effective radius to be $\sim 4$ ". It has been pointed out in the literature (Gadotti 2009) that bulge light dominates the disk light for atleast twice the bulge effective radius. So, it reasonable to expect that to obtain a disk dominated spectrum sufficiently free from any bulge light contamination, one would have to go twice as far (16"). This means we do not have much signal from the disk dominated region to be able to compare the populations of bulges and disks. The metallicity is also near solar which is not too surprising given other features of this galaxy.

This galaxy is clearly quite different from the first two examples in our sample - devoid of star formation and dominated by older stars. It is also different in another major way - it is a barred galaxy. Its formation history is consistent with an old coeaval population but with a small component of recently formed stars. These stars could have formed through gas infall induced by bars.

\subsubsection{NGC 1510}

This galaxy is known to be a part of a pair with the other galaxy being NGC 1512. According to Meurer et al. (2006), this is a pair of galaxies exhibiting starbursts and a common HI envelope. The presence of an intense star burst is confirmed by our spectrum which shows extremely strong $H \alpha$ emission. The age variation as a function of distance from the centre reveals an asymmetric feature. This means that there are more old stars on one side of the galaxy. This population of old stars on one side is also metal rich as can be seen in Figure 6.

These features seem to be a result of the tidal effects of its partner galaxy NGC 1512. The tidal features are clearly visible in NGC 1510 from archival GALEX FUV and NUV images. The tidal interactions have likely led to the asymmetric formation of star burst regions in this galaxy. Again, the $\mathrm{B} / \mathrm{T}$ ratio in this galaxy is quite high at 0.4 .

\subsubsection{IC 2085}

This galaxy is part of NGC 1566 group of galaxies which is a part of the Dorado Group. In comparison to other galaxies in this sample, the spectrum obtained for this galaxy 
Table 3. Best fit StARLight parameters for the spectra from the central regions of the eight galaxies in our sample.

\begin{tabular}{lcccccccccc}
\hline Name & LWZ & MWZ & LWAge & MWAge & Av & Young & Inter & Old & Mass tot & $D_{n}(4000)$ \\
\hline \hline ESO079-007 & 0.007 & 0.009 & $8.275 \pm 0.039$ & $9.706 \pm 0.053$ & $0.488 \pm 0.056$ & 0.768 & 0.0 & 0.232 & 8.429 & 1.076 \\
NGC1522 & 0.006 & 0.007 & $8.018 \pm 0.021$ & $9.324 \pm 0.084$ & $0.314 \pm 0.041$ & 0.849 & 0.063 & 0.088 & 7.715 & 0.983 \\
NGC1510 & 0.006 & 0.008 & $8.029 \pm 0.021$ & $9.356 \pm 0.094$ & $0.282 \pm 0.035$ & 0.754 & 0.194 & 0.052 & 7.917 & 1.002 \\
IC2085 & 0.009 & 0.013 & $8.953 \pm 0.025$ & $9.961 \pm 0.03$ & $0.892 \pm 0.019$ & 0.429 & 0.03 & 0.541 & 8.125 & 1.329 \\
ESO085-030 & 0.008 & 0.01 & $8.627 \pm 0.019$ & $9.82 \pm 0.048$ & $0.582 \pm 0.022$ & 0.597 & 0.0 & 0.403 & 8.285 & 1.201 \\
NGC7709 & 0.006 & 0.01 & $8.066 \pm 0.027$ & $9.697 \pm 0.103$ & $0.116 \pm 0.046$ & 0.871 & 0.0 & 0.129 & 8.581 & 1.011 \\
NGC1533 & 0.02 & 0.02 & $10.176 \pm 0.01$ & $10.224 \pm 0.003$ & $0.042 \pm 0.011$ & 0.018 & 0.0 & 0.982 & 9.065 & 1.731 \\
NGC5750 & 0.011 & 0.015 & $9.615 \pm 0.026$ & $10.053 \pm 0.022$ & $0.279 \pm 0.018$ & 0.171 & 0.0 & 0.829 & 9.358 & 1.543 \\
\hline
\end{tabular}

Notes: Column 1 - The common name of the galaxy; Column 2 - Light Weighted Metallicity; Column 3 - Mass Weighted Metallicity; Column 4 - Log of Light Weighted Age [yr]; Column 5 - Log of Mass Weighted Age [yr]; Column 6 - Extinction Parameter; Column 7 Fraction of Young Stars (Age $<10^{8} \mathrm{yr}$ ); Column 8 - Fraction of Intermediate Stars $\left(10^{8}<\right.$ Age $<10^{9}$ yr); Column 9 - Fraction of Old Stars (Age $>10^{9} \mathrm{yr}$ ); Column $10-$ Log of Galaxy's Total Mass $\left[M_{\odot}\right]$; Column $11-$ The $D_{n}(4000)$ index. The typical uncertainties in case of both light and mass weighted metallicities are of the order of $10^{-4}$ and thus have not been provided in the table above.

is of a relatively lower SNR. Even without performing any modelling, one can see that this galaxy contains both a very old and a very young stellar population in the centre. The presence of strong emission lines such as $H \alpha$ support ongoing star formation as well as recently formed stars while the $4000 \AA$ break caused by several absorption features below $4000 \AA$ is indicative of an older population. Figure 9 shows an asymmetric age gradient around the centre however the stellar metallicity distribution is symmetric. This could be due to the bursts of star formation in the presence of asymmetric dust features. The optical image from Digital Sky Survey (DSS) confirms the presence of disturbed dust lanes.

The best-fit model from STARLIGHT reveals that $50 \%$ of the light is accounted for by a young population with age $<10^{8}$ yr and the rest by very old stars. Such a mixture of very young and very old stars hints at a composite bulge system.

\subsubsection{ESO085-030}

This galaxy shares several features with ESO079-007 except for the star forming regions which are not distributed asymmetrically. About $40 \%$ of the light in the centre of this galaxy is being accounted for by old stars and this fraction falls to $15 \%$ as one moves outwards. The $\mathrm{B} / \mathrm{T}$ ratio as determined from the 3.6-micron imaging is 0.6 with the disk scale length smaller than the bulge effective radius and the Sersic index is highest in the sample. This means that at least in the centre, the bulge which is surrounded by the small disk dominates significantly and thus the trend being observed is due to the bulge. This indicates that pseudobulges grow in a manner similar to disks i.e. inside-out.

That the pseudobulge can have a B/T ratio of 0.6 needs further explanation here. Based on prior studies carried out on pseudobulges, one generally does not expect such a high value of $\mathrm{B} / \mathrm{T}$. These studies have largely focussed on latetype galaxies which are known to be dominated by disks. However, it is possible that in the case of an S0 galaxy, the disk has lowered in luminosity due to a morphology transformation through gas stripping. This would cause an increase in the $\mathrm{B} / \mathrm{T}$ ratio of these objects.

\subsubsection{NGC 5750}

This is an interesting object which exhibits a ring like structure. It is classified as an S0/a galaxy which designates it as a transition class object, i.e. an object which might be speculated to be transforming between S0 and Sa morphology. Sandage, in The Carnegie Atlas of Galaxies speculates that the ring might actually be made of two very tightly bound spiral arms.

The galaxy is barred and in terms of spectral properties, is similar to NGC 1533, comprising of an old population with near solar metallicity. It is also the second barred galaxy in our sample which has been classified as a pseudobulge based on photometric properties, but it shows characteristics that are atypical and not expected of pseudobulges.

\subsubsection{NGC 7709}

All conclusions about the NGC 1522 apply to this galaxy as is - there is evidence of strong ongoing star formation, very little flux contribution from the old stars and an overall subsolar metallicity.

\subsection{Correlations between Photometric and Spectroscopic Parameters}

In Figure 8, are two plots showing the variation of bulge average surface brightness within effective radius against light weighted age and metallicity respectively. We see a clear trend that average surface brightness increases with increasing age and metallicity. All other correlations show significant scatter and thus have not been discussed here. We would like to point out that the present study cannot conclusively determine whether the scatter is because of low number statistics or indicates an absence of a real correlation altogether. Further investigation with a larger sample is warranted.

The Spearman rank correlation coefficient between average surface brightness of the bulge within its effective radius and the logarithm of the light weighted age obtained from the STARLIGHT model, is -0.757 with a significance of $>95 \%$. A similar correlation is seen with metallicity; the rank correlation for this is found to be -0.737 with similar significance. These correlations point at a relation between 

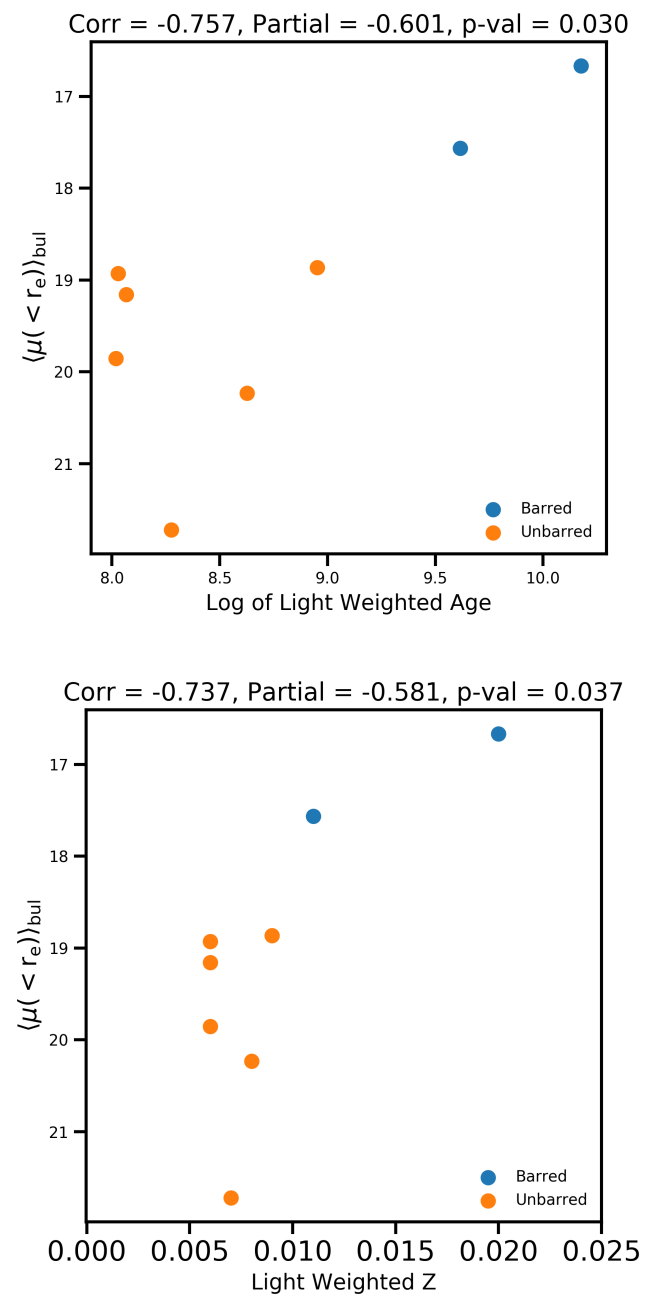

Figure 8. A plot of bulge average surface brightness within effective radius as a function of light weighted age and metallicity.

age and metallicity of the population and the growth / build up of bulges.

These correlations could also be driven by the galaxy mass. For example, if the age or metallicity are correlated with the mass of the galaxy (Gallazzi et al. 2005), then one should expect to see the correlation we find in the upper panel of Figure 8. A simple way to test the possibility is to evaluate the Spearman partial rank correlation between the average surface brightness and age, with the effect of total mass of the galaxy factored out. The masses of our galaxies were determined by a prescription given in Cook et al. (2014) from the M/L ratios at $3.6 \mu \mathrm{m}$. We find that the partial correlation coefficient is -0.601 , which is not significantly different from the Spearman rank correlation coefficient between the two variables, which is -0.757 . Similarly, the partial correlation coefficient between the average surface brightness and metallicity is -0.581 , which is comparable to the correlation coefficient if -0.737 . This seems to suggest that the correlations are not completely driven by the total mass of the galaxy. Note that these correlations hold even if we use the corresponding mass weighted age and metallicity, albeit with a very minor change in the correlation strengths.
Again, we would like to point out that these results are not statistically robust owing to the small number of data points. But the presence of such correlations suggests the need for a study which aims at obtaining both photometric and spectroscopic parameters for a larger sample of galaxies.

\section{DISCUSSION}

We have seen above that the barred galaxies in our sample have largely similar stellar population properties and so is the case with the unbarred galaxies taken as a group but there are important differences between these groups.

\subsection{The unbarred group}

Recall that the parent sample of this study is the sample of 25 pseudobulge hosting S0 galaxies, as described in Vaghmare et al. (2013). The authors classified pseudobulges using a very conservative criterion. Typically, studies aimed at bulge classification adopt a very simple criterion where all bulges with Sérsic index $n<2$ are classified as pseudobulges and others as classical. But as Vaghmare et al. (2013) argue, the parameter $n$ is not easy to constrain and thus there is scope for misclassification. Gadotti (2009) proposed an alternate criterion based on the Kormendy diagram and argued on the merits of such an approach. Vaghmare et al. (2013) adopted a combination of the two criteria to ensure that a secure classification is obtained. The chances of misclassifying a galaxy as a pseudobulge host is thus quite low.

Vaghmare et al. (2015) suggested that the pseudobulge hosting S0s have a lower disk luminosity with respect to their spiral counterparts, which the authors interpret as a disk that has faded while undergoing a transformation in morphology from spiral to S0. The authors argue that this transformation involves stripping of gas which is needed for sustaining active star formation. The signs of active star formation in these galaxies, as indicated by their spectra is in contradiction with this conclusion. Further to confirm if the gas stripping process is a viable scenario, we investigated the environment of these unbarred galaxies. We found that excluding IC 2085, the galaxies in the unbarred group are in an isolated environment.

One possible resolution is that these isolated galaxies are not gas stripped spirals. In this case, we have to conclude that these disks are inherently less luminous and somehow such disks have an affinity to be a part of pseudobulge hosting S0 galaxies. Another explanation is that these objects are gas stripped spirals but have had their gas replenished through numerous gas rich minor mergers which in turn led to complex star formation (Penoyre et al. 2017).

\subsection{The barred group}

An interesting commonality in the two galaxies in this group, apart from the stellar population, is that these galaxies are barred. With the usual caveat of lack of robustness due to small numbers, this common observation allows for some speculation on why, despite such a conservative classification criterion, the bulges of these galaxies are found not to be consistent with pseudobulges. Recall that the photometric 
parameters of the bulges as derived by performing 2 -d bulgedisk decomposition, have been used for classifying them. One may thus argue that the presence of a bar has a systematic effect on the derived bulge properties (Laurikainen et al. 2005; Gadotti 2009). But Vaghmare et al. (2013) recognize this problem and take care to fit a bar component as well. Thus the unaccounting of a bar component cannot explain this observation.

Another explanation for the old stellar population in these galaxies is as follows. The presence of the bar led to an amplified funnelling of gas towards the centre of the galaxies in the past. This allowed the pseudobulge to form much earlier than the rest of the galaxies where there was no bar component to aid the quick formation of a pseudobulge. As a result the pseudobulges in both these galaxies contain a very old population as can be seen in the Figure 4 . In the case of NGC 1533, there does not seem to have been any gas available to lead to recent star formation while in the case of NGC 5750, there is some evidence of recent star formation. A confirmation of whether the bar action can explain the older age could be obtained from a high spatial resolution rotation curve for these galaxies. The relative domination of a random component of stellar velocities vs systematic rotation may be able to shed light on whether there is funnelling of gas taking place. However, such a study is not possible using the present data.

\section{STELLAR POPULATIONS IN PSEUDOBULGE HOSTING SOS - BARRED VS UNBARRED}

In section 5.2, we saw that the only pseudobulge hosting S0 galaxies which exhibited a significant departure from the overall nature of star formation history observed among other galaxies were barred. However, with such low numbers, it is not appropriate to firmly conclude that the nature of pseudobulges in S0 galaxies is a function of the presence or absence of a bar. But it is reasonable to expect a difference since we know that the bar does affect the nature of stellar orbits and gas dynamics in a galaxy. Further, a bar is known to be a driver of growth in bulges in the secular evolution scenario.

We thus decided to perform a quick analysis to see if such a difference between pseudobulges in barred S0s and unbarred S0s, is indeed real. Mishra et al. (2017) have presented evidence for the bimodal stellar age distribution of pseudo-bulges of unbarred S0 galaxies as probed by the $D_{n}(4000)$ index. This index is a reliable indicator of the mean age of galaxy stellar population which is quantified using the strength of the $4000 \AA$ spectral break arising due to the accumulation of absorption lines of metals in the atmosphere of old, low-mass stars in galaxies. The light-weighted mean stellar ages of $\sim 2$ Gyr corresponds to $D_{n}(4000)$ index of 1.5 (Kauffmann et al. 2003) and using this Mishra et al. (2017) have divided the sample of unbarred pseudo-bulges in S0 galaxies with a young (having $\left.D_{n}(4000)<1.5\right)$ and old (having $\left.D_{n}(4000) \geq 1.5\right)$ stellar populations. In their study, Mishra et al. (2017) did not use S0 galaxies that host a bar. In this work, we use the sample and data described in Mishra et al. (2017) but for pseudo-bulges in S0 galaxies that host a bar.

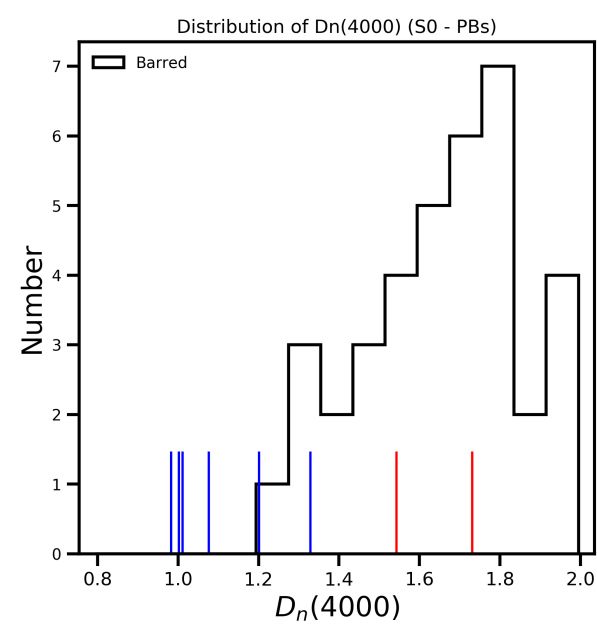

Figure 9. A plot showing the distribution of $D_{n}(4000)$ index for barred pseudobulge hosting S0 galaxies from the sample used in Mishra et al. (2017). The blue vertical lines represent the $D_{n}(4000)$ index for unbarred galaxies in our sample while the red vertical lines represent the barred galaxies.

In Figure 9, we show the distribution of the $D_{n}(4000)$ index for all the barred S0s with pseudobulge from the sample in Mishra et al. (2017). This figure reveals that the barred galaxies preferentially have $D_{n}(4000) \geq 1.5$ ( older stellar populations), which is consistent with our current study. The figure also shows the $D_{n}(4000)$ index for the eight galaxies in our sample. Our current spectroscopic analysis seems to suggest that the bimodality presented in Mishra et al. (2017) could be caused by the presence of a bar. This, in turn, suggests that pseudobulges, when found in barred S0s exhibit a dominant older stellar population while an unbarred pseudobulge hosting S0 can exhibit significant contribution from both young and old populations. Our current spectroscopic sample, however, does not have pseudobulge hosting S0s which have a very old population and do not contain a bar. Commenting on the exact nature of such differences in S0s with pseudobulge however, is rather difficult.

\section{SUMMARY}

In this paper, we have studied eight S0 galaxies established by Vaghmare et al. (2015) to host pseudobulges, using longslit spectra obtained from the SALT-RSS. We have modelled the spectra using STARLIGHT to derive detailed star formation histories of these galaxies as well as age and metallicity gradients. The present study focusses primarily on the spectra of the central regions of the galaxy and, using the spectral information, confronts the conclusions drawn by Vaghmare et al. (2015).

The most important conclusion is that these eight galaxies are not all similar in terms of their star formation histories. This means that objects grouped in one class using photometric criteria (the class here being pseudobulge hosting S0s) can exhibit diverse properties. The present study brings out the need for further studies that are based on spectral analysis to truly constrain the formation mechanisms at work in the formation of these objects. 
We discovered that six of these eight galaxies, which are unbarred, exhibit a complex star formation history with active star formation. We attempt reconciling the observed ongoing star formation with the conclusion reached by Vaghmare et al. (2015) that these are gas stripped spirals. It has been suggested by Poggianti et al. (2017) that the process of ram pressure stripping in galaxies can cause initial fuelling of gas inwards, which could lead to star formation in the central regions. Once the gas is stripped, the disk can fade, but residual gas in the central region could be responsible for the observed star formation. This would make fading due to stripping consistent with our observations. But since most of the galaxies are in an isolated environment the question would remain as to what caused the stripping.

In the other two galaxies, which are barred, we find a population similar to those expected in elliptical galaxies. We have detected a trace of recent star formation in one of these galaxies which likely indicates that the bar is funnelling / has funnelled gas towards the center leading to a formation of a younger group of stars. We have also used a sample of SDSS galaxies to show that the preferential occurrence of older populations in barred S0 galaxies, suggested by the spectral data, is likely a real phenomenon. The study of this SDSS sample also suggests that there are unbarred pseudobulge hosting S0s with very old populations (Mishra et al. 2017). However, no such object has been found in the present study.

Using the data obtained from the SALT-RSS, it is possible to perform a very detailed analysis of the star formation history of the off-center regions of the galaxy. This can lead to new insights into how galaxies formed. We defer such a detailed study to a future paper.

\section{ACKNOWLEDGEMENTS}

We thank the anonymous referee for insightful comments that have improved both the content and presentation of this paper. All of the observations reported in this paper were obtained with the Southern African Large Telescope (SALT) under program 2014-1-IUCAA-RSA-OTH-001 (PI: A.K. Kembhavi). KV would like to acknowledge Alexei Kniazev, Steve Crawford and R Srianand for useful discussions and guidance. KV also acknowledges the Council of Scientific and Industrial Research (CSIR), India for financial assistance and SAAO \& SALT for travel and hospitality support. Part of this work for KV was possible due to a grant from the National Knowledge Network (NKN), India. $\mathrm{SB}$ acknowledges SAAO where part of this work has been done. PV acknowledges support from the SA National Research Foundation. YW thanks IUCAA for hosting him on his sabbatical where a part of this work was done. We acknowledge support from a South African National Research Foundation grant (PID-93727) and from a bilateral grant under the Indo-South Africa Science and Technology Cooperation (PID-102296) funded by the Department of Science and Technology (DST) of the Indian and South African governments. PyRAF is a product of the Space Telescope Science Institute, which is operated by AURA for NASA and was extensively utilised for the spectral data reduction pipelines.

\section{REFERENCES}

Aguerri J. A. L., 2012, Advances in Astronomy, 2012, 382674

Aragón-Salamanca A., Bedregal A. G., Merrifield M. R., 2006, A\&A, 458, 101

Balogh M. L., Morris S. L., Yee H. K. C., Carlberg R. G., Ellingson E., 1999, ApJ, 527, 54

Barway S., Kembhavi A., Wadadekar Y., Ravikumar C. D., Mayya Y. D., 2007, ApJ, 661, L37

Barway S., Wadadekar Y., Kembhavi A. K., Mayya Y. D., 2009, MNRAS, 394, 1991

Barway S., Wadadekar Y., Vaghmare K., Kembhavi A. K., 2013, MNRAS, 432, 430

Bekki K., Couch W. J., 2011, MNRAS, 415, 1783

Buckley D. A. H., Swart G. P., Meiring J. G., 2006, in Proc. SPIE, Vol. 6267, Instrument Design and Performance for Optical/Infrared Ground-based Telescopes, p. $62670 \mathrm{Z}$

Burgh E. B., Nordsieck K. H., Kobulnicky H. A., Williams T. B., O'Donoghue D., Smith M. P., Percival J. W., 2003, in Proc. SPIE, Vol. 4841, Instrument Design and Performance for Optical/Infrared Ground-based Telescopes, Iye M., Moorwood A. F. M., eds., pp. 1463-1471

Cid Fernandes R., Mateus A., Sodré L., Stasińska G., Gomes J. M., 2005, MNRAS, 358, 363

Cook D. O., Dale D. A., Johnson B. D., Van Zee L., Lee J. C., Kennicutt R. C., Calzetti D., Staudaher S. M., Engelbracht C. W., 2014, MNRAS, 445, 899

Crawford S. M., Still M., Schellart P., Balona L., Buckley D. A. H., Dugmore G., Gulbis A. A. S., Kniazev A., Kotze M., Loaring N., Nordsieck K. H., Pickering T. E., Potter S., Romero Colmenero E., Vaisanen P., Williams T., Zietsman E., 2010, in Society of Photo-Optical Instrumentation Engineers (SPIE) Conference Series, Vol. 7737, Society of Photo-Optical Instrumentation Engineers (SPIE) Conference Series, p. 25

de Vaucouleurs G., de Vaucouleurs A., Corwin Jr. H. G., Buta R. J., Paturel G., Fouqué P., 1991, Third Reference Catalogue of Bright Galaxies. Volume I: Explanations and references. Volume II: Data for galaxies between $0^{h}$ and $12^{h}$. Volume III: Data for galaxies between $12^{h}$ and $24^{h}$.

Dressler A., 1980, ApJ, 236, 351

Falcón-Barroso J., Bacon R., Bureau M., Cappellari M., Davies R. L., Emsellem E., Krajnović D., Kuntschner H., McDermid R., Peletier R. F., de Zeeuw P. T., 2004, Astronomische Nachrichten, 325, 92

Fisher D. B., Drory N., 2008, AJ, 136, 773

Gadotti D. A., 2009, MNRAS, 393, 1531

Gallazzi A., Charlot S., Brinchmann J., White S. D. M., Tremonti C. A., 2005, MNRAS, 362, 41

Gunn J. E., Gott J. Richard I., 1972, ApJ, 176, 1

Just D. W., Zaritsky D., Sand D. J., Desai V., Rudnick G., 2010, ApJ, 711, 192

Kauffmann G., Heckman T. M., White S. D. M., Charlot S., Tremonti C., Brinchmann J., Bruzual G., Peng E. W., Seibert M., Bernardi M., Blanton M., Brinkmann J., Castander F., Csábai I., Fukugita M., Ivezic Z., Munn J. A., Nichol R. C., Padmanabhan N., Thakar A. R., Weinberg D. H., York D., 2003, MNRAS, 341, 33

Kobulnicky H. A., Nordsieck K. H., Burgh E. B., Smith M. P., Percival J. W., Williams T. B., O'Donoghue D., 2003, in Proc. SPIE, Vol. 4841, Instrument Design and Performance for Optical/Infrared Ground-based Telescopes, Iye M., Moorwood A. F. M., eds., pp. 1634-1644

Kormendy J., Kennicutt Jr. R. C., 2004, ARA\&A, 42, 603

Laurikainen E., Salo H., Buta R., 2005, MNRAS, 362, 1319

Laurikainen E., Salo H., Buta R., Knapen J. H., Comerón S., 2010, MNRAS, 405, 1089

Maltby D. T., Aragón-Salamanca A., Gray M. E., Hoyos C., Wolf C., Jogee S., Böhm A., 2015, MNRAS, 447, 1506 
Mapelli M., Moore B., Bland-Hawthorn J., 2008, MNRAS, 388, 697

Mapelli M., Rampazzo R., Marino A., 2015, A\&A, 575, A16

Mazzei P., Marino A., Rampazzo R., Galletta G., Bettoni D., 2014, Advances in Space Research, 53, 950

Meurer G. R., Hanish D. J., Ferguson H. C., Knezek P. M., Kilborn V. A., Putman M. E., Smith R. C., Koribalski B., Meyer M., Oey M. S., Ryan-Weber E. V., Zwaan M. A., Heckman T. M., Kennicutt Jr. R. C., Lee J. C., Webster R. L., BlandHawthorn J., Dopita M. A., Freeman K. C., Doyle M. T., Drinkwater M. J., Staveley-Smith L., Werk J., 2006, ApJS, 165,307

Mishra P. K., Barway S., Wadadekar Y., 2017, MNRAS, 472, L89

Peng C. Y., Ho L. C., Impey C. D., Rix H.-W., 2010, AJ, 139, 2097

Penoyre Z., Moster B. P., Sijacki D., Genel S., 2017, MNRAS, 468, 3883

Poggianti B. M., Jaffé Y. L., Moretti A., Gullieuszik M., Radovich M., Tonnesen S., Fritz J., Bettoni D., Vulcani B., Fasano G., Bellhouse C., Hau G., Omizzolo A., 2017, Nature, 548, 304

Querejeta M., Eliche-Moral M. C., Tapia T., Borlaff A., Rodríguez-Pérez C., Zamorano J., Gallego J., 2015, A\&A, 573, A78

Renzini A., 1999, in The Formation of Galactic Bulges, Carollo C. M., Ferguson H. C., Wyse R. F. G., eds., p. 9

Richards J. W., Freeman P. E., Lee A. B., Schafer C. M., 2009, MNRAS, 399, 1044

Rizzo F., Fraternali F., Iorio G., 2018, MNRAS, 476, 2137

Sánchez-Blázquez P., 2016, Galactic Bulges, 418, 127

Sánchez-Blázquez P., Peletier R. F., Jiménez-Vicente J., Cardiel N., Cenarro A. J., Falcón-Barroso J., Gorgas J., Selam S., Vazdekis A., 2006, MNRAS, 371, 703

Tapia T., Eliche-Moral M. C., Aceves H., Rodríguez-Pérez C., Borlaff A., Querejeta M., 2017, A\&A, 604, A105

Vaghmare K., Barway S., Kembhavi A., 2013, ApJ, 767, L33

Vaghmare K., Barway S., Mathur S., Kembhavi A. K., 2015, MNRAS, 450, 873

van Dokkum P. G., 2001, PASP, 113, 1420

Vulcani B., Poggianti B. M., Moretti A., Mapelli M., Fasano G., Fritz J., Jaffé Y., Bettoni D., Gullieuszik M., Bellhouse C., 2018, ApJ, 852, 94

Wilman D. J., Oemler Jr. A., Mulchaey J. S., McGee S. L., Balogh M. L., Bower R. G., 2009, ApJ, 692, 298

\section{APPENDIX A}

As mentioned in Section 3, we construct the base spectra starting from a collection of 150 SSP spectra from the MILES library. These spectra are then input to the algorithm proposed by Richards et al. (2009) and a new set of 45 base spectra are obtained. Table 4 shows the ages and the metallciites of the final base spectra used with STARLIGHT.
Table 4. Ages and metallicities of the base spectra used in the present study.

\begin{tabular}{|c|c|c|}
\hline S.No. & Age (yr) & Metallicity \\
\hline 1 & $2.6396 \times 10^{8}$ & $6.8388 \times 10^{-3}$ \\
\hline 2 & $1.3111 \times 10^{10}$ & $1.3994 \times 10^{-2}$ \\
\hline 3 & $9.7615 \times 10^{9}$ & $1.0982 \times 10^{-2}$ \\
\hline 4 & $2.6210 \times 10^{8}$ & $1.0380 \times 10^{-2}$ \\
\hline 5 & $8.9564 \times 10^{8}$ & $1.0630 \times 10^{-2}$ \\
\hline 6 & $5.6230 \times 10^{8}$ & $1.0638 \times 10^{-2}$ \\
\hline 7 & $4.8752 \times 10^{8}$ & $8.9622 \times 10^{-3}$ \\
\hline 8 & $8.9130 \times 10^{8}$ & $4.0000 \times 10^{-3}$ \\
\hline 9 & $7.9791 \times 10^{7}$ & $5.9980 \times 10^{-3}$ \\
\hline 10 & $1.8109 \times 10^{9}$ & $1.0634 \times 10^{-2}$ \\
\hline 11 & $6.1258 \times 10^{9}$ & $6.6698 \times 10^{-3}$ \\
\hline 12 & $1.2589 \times 10^{10}$ & $2.0000 \times 10^{-2}$ \\
\hline 13 & $7.3106 \times 10^{9}$ & $1.5997 \times 10^{-2}$ \\
\hline 14 & $5.4542 \times 10^{9}$ & $8.9952 \times 10^{-3}$ \\
\hline 15 & $9.3783 \times 10^{7}$ & $1.0016 \times 10^{-2}$ \\
\hline 16 & $7.0790 \times 10^{8}$ & $2.0000 \times 10^{-2}$ \\
\hline 17 & $7.3706 \times 10^{9}$ & $9.9992 \times 10^{-3}$ \\
\hline 18 & $7.5109 \times 10^{8}$ & $6.0003 \times 10^{-3}$ \\
\hline 19 & $4.2302 \times 10^{9}$ & $8.8058 \times 10^{-3}$ \\
\hline 20 & $3.9137 \times 10^{9}$ & $6.0026 \times 10^{-3}$ \\
\hline 21 & $7.9430 \times 10^{8}$ & $8.0000 \times 10^{-3}$ \\
\hline 22 & $2.9023 \times 10^{9}$ & $9.3242 \times 10^{-3}$ \\
\hline 23 & $2.1840 \times 10^{8}$ & $5.3372 \times 10^{-3}$ \\
\hline 24 & $1.0052 \times 10^{9}$ & $1.0613 \times 10^{-2}$ \\
\hline 25 & $1.4125 \times 10^{10}$ & $2.0000 \times 10^{-2}$ \\
\hline 26 & $1.4844 \times 10^{9}$ & $1.0627 \times 10^{-2}$ \\
\hline 27 & $7.4950 \times 10^{9}$ & $9.0092 \times 10^{-3}$ \\
\hline 28 & $3.3522 \times 10^{9}$ & $1.2004 \times 10^{-2}$ \\
\hline 29 & $1.1220 \times 10^{10}$ & $2.0000 \times 10^{-2}$ \\
\hline 30 & $6.5028 \times 10^{8}$ & $8.9704 \times 10^{-3}$ \\
\hline 31 & $1.3282 \times 10^{8}$ & $1.8674 \times 10^{-2}$ \\
\hline 32 & $1.7903 \times 10^{9}$ & $1.3993 \times 10^{-2}$ \\
\hline 33 & $3.9982 \times 10^{8}$ & $5.3293 \times 10^{-3}$ \\
\hline 34 & $1.7893 \times 10^{9}$ & $6.0078 \times 10^{-3}$ \\
\hline 35 & $1.6733 \times 10^{10}$ & $2.0000 \times 10^{-2}$ \\
\hline 36 & $3.2165 \times 10^{8}$ & $8.9766 \times 10^{-3}$ \\
\hline 37 & $1.0998 \times 10^{10}$ & $1.4071 \times 10^{-2}$ \\
\hline 38 & $2.3371 \times 10^{9}$ & $8.9891 \times 10^{-3}$ \\
\hline 39 & $3.2452 \times 10^{9}$ & $6.0036 \times 10^{-3}$ \\
\hline 40 & $6.3100 \times 10^{7}$ & $5.9987 \times 10^{-3}$ \\
\hline 41 & $1.1220 \times 10^{9}$ & $2.0000 \times 10^{-2}$ \\
\hline 42 & $1.4188 \times 10^{9}$ & $5.3253 \times 10^{-3}$ \\
\hline 43 & $1.0286 \times 10^{10}$ & $1.2000 \times 10^{-2}$ \\
\hline 44 & $2.0716 \times 10^{8}$ & $1.3105 \times 10^{-2}$ \\
\hline 45 & $1.1273 \times 10^{9}$ & $1.0638 \times 10^{-2}$ \\
\hline
\end{tabular}

Notes: The base spectra whose ages and metallicities are given above have been constructed by inputting 150 initial base spectra from the MILES library to the MATLAB code which implements the algorithm by Richards et al. (2009). 